\title{
Peso al nacimiento de abejas reina de la raza Apis mellifera iberiensis
}

\author{
Padilla, F. ${ }^{@}$; Cabanes, F.; Montilla, I. y Flores J.M.
}

Departamento de Zoología. Universidad de Córdoba. Córdoba. España.

\section{PALABRAS CLAVE}

Abejas.

Apicultura.

Cría de reinas.

Producción de reinas.

\section{RESUMEN}

En el mundo apícola se acepta que la producción de una determinada colonia de abejas se encuentra directamente relacionada con la calidad de la reina. De todas las posibles variables relacionadas con dicha calidad, el peso al nacimiento se considera una de las más útiles. En nuestro trabajo hemos estudiado el efecto de la colonia criadora de la subespecie local de abejas (Apis melífera iberiensis Engel), sobre el peso al nacimiento de las nuevas reinas, y de las celdillas en las que fueron criadas. Para realizar la cría hemos utilizado el método Doolittle; este procedimiento se encuentra ampliamente difundido y es empleado de forma habitual por los productores. Entre los meses de marzo y mayo de 2019 criamos 140 reinas, realizando ocho ciclos de cría y utilizado cuatro colmenas criadoras diferentes. El peso medio de las reinas al nacimiento fue de $194 \pm 26 \mathrm{mg} \mathrm{(x \pm SD)}$, y el de las celdillas de cría $604 \pm 125$ ( $x \pm$ SD). Los resultados obtenidos no mostraron diferencias significativas entre las colmenas criadoras, para la variable peso de las reinas al nacimiento, pero si para la variable peso de las celdillas reales.

Weight at emergence of honeybee queens of the Apis mellifera iberiensis breed

\section{ADDITIONAL KEYWORDS}

Beekeeping.

Breeding.

Honeybees.

Queen production.

Queen rearing.

\section{INFORMATION}

\section{Cronología del artículo.}

Recibido/Received: 27.10.2020

Aceptado/Accepted: 20.11.2020

On-line: 15.04.2021

Correspondencia a los autores/Contact e-mail:

padilla@uco.es

\section{SUMMARY}

In the practice of beekeeping it is generally accepted that the colony production is directly related to the quality of the queen. Of all the possible variables related to such quality, birth weight is considered one of the most useful. In our study we have looked the effect of the breeding colony of the local bee subspecies (Apis melifera iberiensis Engel), on the weight of the new queens at birth, and the weight of the wax cells in which they were reared. To carry out the breeding we have used the Doolittle method, a procedure widely used by beekeepers. Between the months of March and May of 2019, we raised 140 queens, carrying out eight breeding cycles and using four different hives. The mean weight of the queens at birth was $194 \pm 26$ $\mathrm{mg}(\mathrm{x} \pm \mathrm{SD})$, and that of the brood cells $604 \pm 125(\mathrm{x} \pm \mathrm{SD})$. The results obtained did not show significant differences between the brood hives, for the weight variable of the queens at birth, but there were differences in the case of the variable weight of the queen cells.

\section{INTRODUCCIÓN.}

El uso de reinas de calidad es un prerrequisito o condición necesaria para obtener una alta producción de las colonias de abejas (Apis mellifera) (Kahya et al. 2008). Se encuentra ampliamente aceptado el hecho de que el peso al nacimiento es un indicador de la calidad de estos insectos. De hecho, algunos criadores europeos consideran que, en el caso de reinas criadas artificialmente, un peso al nacimiento inferior a 190 mg es indicativo de una baja calidad. Este indicador de calidad está condicionado por diferentes factores, siendo los más importantes: la genética, la época del año en la que se realiza la cría, la edad de la larva utilizada para producir las nuevas reinas, o la colmena criadora utilizada (Mirza et al. 1967; Okuyan et al. 2018; Woyke, 1971).

Se admite que la calidad de una reina puede ser valorada midiendo características cuantitativas tales como: peso en vivo de los animales, peso de los ovarios, número de ovariolas, número de espermatozoides almacenados en la espermateca, tiempo transcurrido desde el nacimiento hasta el inicio de la puesta, o número de huevos puestos en 24 h. (Delaney et al. 2010; Hatjina et al. 2014).

Los apicultores consideran que una reina de calidad, además de ser grande y presentar un buen aspecto, debe de expresar a través de su descendencia otras características deseables, tales como: alta fecundidad y 
consecuentemente capacidad de mantener una alta población de obreras, alto comportamiento higiénico, que incluye la resistencia a las enfermedades, alta producción de miel y/o polen, baja tendencia de enjambrazón y baja agresividad.

En el presente trabajo hemos estudiado el peso al nacimiento de abejas reina de la raza A. m. iberiensis con el objetivo de conocer el valor de esta variable para esta raza. También hemos estudiado el peso de la celdilla de cría, como una característica relacionada con el tamaño y peso de la reina, asumiendo la posible existencia de una correlación entre estas dos variables.

\section{MATERIAL Y MÉTODOS.}

Los ensayos fueron realizados en el colmenar experimental situado en el Campus de Rabanales de la

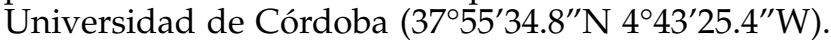
Entre los meses de marzo y mayo de 2019 se realizaron 8 ciclos de cría (ensayos) utilizando el método Doolittle. El fundamento de este método es reconducir el desarrollo de una larva, inicialmente destinada a ser obrera, trasvasándola a una celdilla artificial de tamaño y disposición propios de una celdilla real.

Para realizar los ensayos utilizamos cuatro colmenas tipo Langstroth con un cuerpo y un alza (20 cuadros). Entre el cuerpo y el alza se colocó un excluidor de reinas, manteniendo la reina de la colmena en el cuerpo, y utilizando el alza para la cría de las nuevas reinas. Como sustituto de las celdillas reales usamos cúpulas fabricadas con cera, sujetas en soportes portacúpulas. Los soportes eran fijados en listones y estos en número de dos, eran situados horizontalmente en marcos de panales sin cera, dispuestos de tal forma que las cúpulas quedaban en posición vertical. Cada listón contaba con 15 cúpulas, por lo tanto, en cada ciclo se criaron 30 reinas/colmena.

Todas las larvas utilizadas en cada serie experimental tenían entre 1 y 2 días de edad, eran hermanas y procedían de la misma colonia donadora. Se utilizaron diferentes colonias donantes en los diferentes ensayos. Una vez que las celdas reales eran operculadas por las nodrizas, se trasladaban los listones a una estufa con una temperatura de $35^{\circ} \mathrm{C}$ y $80 \%$ de humedad.

A los 11-12 días después de haber realizado el traslarve se inician los nacimientos de las reinas. Se realizaron controles periódicos durante 2 días, hasta que finalizaron los nacimientos de todos los insectos viables. Una vez nacidos se pesaban los animales, y las celdillas en las que habían sido criados.

Para el estudio estadístico de los datos se utilizó el programa SPSS Statistics versión 17.0. Debido a que parte de los datos obtenidos no presentaban una distribución normal, se emplearon estadísticos no paramétricos.

\section{RESULTADOS.}

En la cría artificial de abejas reina, para conocer el nivel de éxito de cada ciclo de cría, es importante valorar el porcentaje de larvas aceptadas y criadas por
Tabla I. Porcentajes totales de lavas trasvasadas aceptadas y reinas nacidas en cada ciclo de cría (Total percentages of accepted larvae and queens born in each rearing cycle).

\begin{tabular}{lll}
\hline Fecha de traslarve & Larvas aceptadas & Reinas nacidas \\
$21 / 03 / 2019(1)$ & $63,33 \%$ & $94,73 \%$ \\
$28 / 03 / 2019(2)$ & $83,33 \%$ & $100,00 \%$ \\
$04 / 04 / 2019(3)$ & $76,66 \%$ & $91,30 \%$ \\
$25 / 04 / 2019(4)$ & $40,00 \%$ & $100,00 \%$ \\
$02 / 05 / 2019(5)$ & $53,33 \%$ & $75,00 \%$ \\
$09 / 05 / 2019(6)$ & $30,00 \%$ & $100,00 \%$ \\
$16 / 05 / 2019(7)$ & $60,00 \%$ & $94,44 \%$ \\
$23 / 05 / 2019(8)$ & $73,33 \%$ & $100,00 \%$ \\
Total & $60,00 \%$ & $94,44 \%$ \\
\hline
\end{tabular}

cada colonia, así como el número de reinas nacidas. En la Tabla I se muestran los porcentajes de aceptación, así como el número de insectos nacidos en cada ciclo o ensayo. El nivel de aceptación fue del 60\% y el número de animales nacidos, en relación a los aceptados, fue del $94,44 \%$.

En la Tabla II se resumen los pesos medios de las reinas y las celdas reales en las que fueron criadas, para cada colmena en cada ensayo. El peso medio de las reinas teniendo en cuenta todos los ciclos de cría realizados fue de $194 \pm 0,26 \mathrm{mg}$ y el de las celdillas de cría de $604 \pm 125 \mathrm{mg}$.

El análisis estadístico de los resultados lo iniciamos con la aplicación de la prueba de normalidad de Kolmogórov-Smirnof. Los resultados mostraron que, en el caso del peso de los animales, el valor de significación era inferior a 0,005 (no se ajusta a una distribución normal), pero en el caso del peso de las celdillas de cera el valor de significación fue de 0,054.

Basándonos en estos resultados, decidimos aplicar pruebas no paramétricas para estudiar la posible existencia de diferencias significativas, entre colmenas o entre ensayos (ciclos de cría).

Cuando aplicamos la prueba de Kruskal-Wallis (KW) teniendo en cuenta la colmena criadora, la prueba de contraste de hipótesis para la variable peso de la reina halló un valor de " $\mathrm{p}$ " de 0,069 , por lo tanto, aceptamos la hipótesis nula, es decir, la no existencia de diferencias significativas. La aplicación de la misma prueba a la variable peso de la celdilla mostró un valor de " $\mathrm{p}$ " inferior a 0,05 , por lo que se rechazó la hipótesis nula. La prueba K-W a posteriori confirmó la existencia de dos subconjuntos homogéneos, uno que incluía una única colmena (la $n^{\circ} 951$ ), y otro formado por las tres restantes ( $n^{\circ} 215, n^{\circ} 326$ y no 991). En la Figura 1 se muestran los pesos medios de las reinas y celdillas teniendo en cuenta la colmena criadora, resultando evidente que las abejas de la colmena 951 fueron las que fabricaron las celdillas reales de mayor peso. 


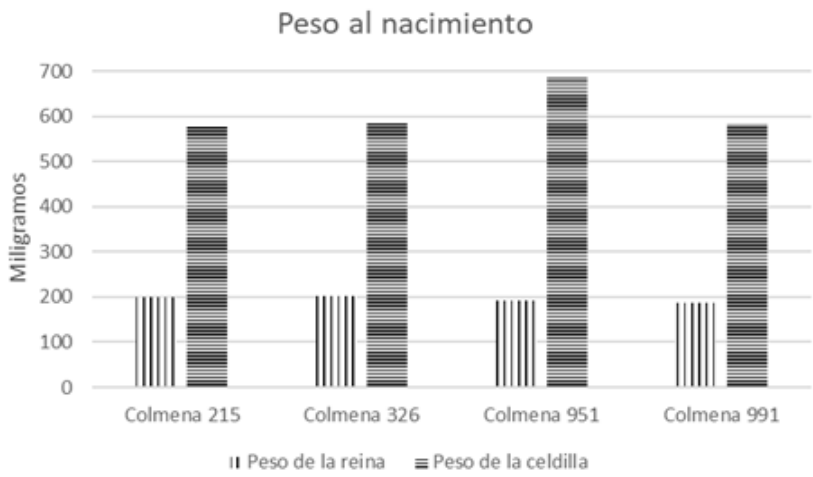

Figura 1. Peso medio al nacimiento de las abejas reina y sus celdas de cría (Average birth weight of queen bees and their brood cells).

Si en lugar de considerar la colmena criadora, consideramos la fecha en la que se realizaron los ensayos, la aplicación de la prueba K-W a las variables peso de las reinas al nacimiento y peso de las celdillas, mostró en los dos casos un valor de " $\mathrm{p}$ " inferior a 0,05 . Por lo tanto, podemos afirmar que existen diferencias significativas entre colmenas con un nivel de significación del $5 \%$. De estos análisis se excluyó el ensayo $4^{\circ}$, ya que en dos de las colmenas no se aceptaron ninguna de las larvas trasvasadas.

Para la variable peso de la reina al nacimiento la prueba K-W a posteriori mostró la existencia de dos subconjuntos, uno formado por los ensayos 2, 5, 6 y 7, que incluía las fechas en las que el peso medio de los insectos fue menor; y otro formado por los ensayos 1 , 3 y 8 que incluía a los de mayor peso. En el caso de la variable peso de la celdilla de cría se obtuvieron tres grupos. Uno formado por los ensayos 7 y 8 (celdillas de menor peso), otro que incluía los ensayos 2,5 y 6 (celdillas de peso medio) y el tercero sólo incluía el ensayo 1 (celdillas de mayor peso).

Finalmente aplicamos el mismo análisis, pero teniendo en cuenta cada ensayo de forma independiente; de este análisis se excluyó el ensayo 4 por los mismos motivos expuestos anteriormente. En el caso de la variable peso de la reina, no se encontraron diferencias significativas entre colmenas dentro de cada ensayo. Pero en el caso del peso de la celdilla de cría, si existían diferencias significativas en los ensayos 1, 2, 3 y 8. La prueba $\mathrm{K}-\mathrm{W}$ a posteriori mostro en cada ensayo dos diferentes subconjuntos homogéneos; en los ensayos 2 y 8 la colmena 951 era el único integrante de uno de los subconjuntos.

\section{DISCUSIÓN}

Desde un punto de vista práctico el comportamiento reproductivo de una colonia de abejas, puede ser considerado, al menos en parte, como una ampliación del fenotipo de la reina (Rangel et al. 2012). Son variados los factores, tanto ambientales como genéticos, que lo condicionan (Al-Ghzawi \& Zaitoun, 2008). De hecho, los apicultores productores de reinas asumen que existen colmenas buenas y malas donantes y/o criadoras.
En nuestros ensayos la tasa de aceptación media, es decir, la proporción de larvas que son criadas por unas determinadas colonias, en relación al número de larvas trasplantadas, fue del $60 \%$. Mohammedi \& Le Conte (2000) realizan series de cría en dos épocas del año que definen como periodo favorable (abril-junio) y desfavorable (diciembre-febrero), obteniendo una tasa de aceptación en la época favorable de entre el 41 y 52\% y en la desfavorable de entre el 23 y $32 \%$. Wilkinson \& Brown (2002) en su trabajo sobre cría de reinas, citan una tasa media de aceptación del $81 \%$, muy superior a la obtenida por nosotros. Pero si en lugar de usar la aceptación media total, consideramos la obtenida en cada ciclo de cría, los valores obtenidos por estos autores varían entre el 25 y 100\%. En nuestro caso los valores obtenidos se situaron entre el 36,8 y 83,3\%.

El hecho de que la tasa de aceptación sea relativamente variable, puede ser explicada atendiendo a diferentes agentes causales. Usar el método Doolittle implica manipular larvas de corta edad, seres vivos de pequeño tamaño y muy delicados, que hay que extraer de una celdilla y depositar en una cúpula (celdilla real artificial), sin que sufran ningún tipo daño, o sean sometidas a un estrés excesivo. En nuestro caso se puso especial énfasis en la correcta manipulación de las larvas, además en todas las series de cría fue la misma persona la encargada de realizar el traslarve. En el caso de que minimicemos los posibles daños originados en el proceso de manipulación, tendremos que buscar el fundamento de esta variabilidad en la biología de la colonia, y/o las condiciones ambientales bajo las que se desarrolló cada ensayo (Mohammedi \& Le Conte 2000; Sagili y cols. 2018, Cengiz et al. 2019).

Kruk \& Skowronek (2002) estudian el efecto de diferentes factores ambientales sobre la eficiencia de la cría artificial de reinas. Encuentran que la mayor tasa de aceptación se produce en el mes de mayo (55,8\%) y la mínima en el de julio (25,8\%). Además, citan que factores tales como la temperatura del aire, la radiación solar o la humedad relativa, tenían un ligero, pero significativo efecto sobre los resultados. También el flujo de néctar, es decir, el nivel de disponibilidad y recolección de este recurso influyó sobre los resultados, obteniéndose una mayor aceptación cuando el mismo era moderado, teniendo un efecto negativo tanto la alta como la baja tasa de recolección de este recurso.

El peso al nacimiento se considera un indicador de calidad, ya que se presupone determinado por las condiciones en las que se ha realizado la cría (ej. tamaño de la celdilla, cuidados dispensados, o nutrición de las larvas), y el genotipo de los animales (Skowronek et al. 2004). Winston (1991, p. 54) cita que los valores medios de los pesos de las reinas, de acuerdo a 17 diferentes estudios realizados, se situaban entre los 178 y 292 mg. Skowronek et al. (2004) estudian la evolución del peso de las reinas de la raza $A$. m. carnica desde el nacimiento hasta que los insectos inician la puesta de huevos, encontrando un peso medio al nacimiento de 226,71 $\pm 21,72 \mathrm{mg}(\overline{\mathrm{X}} \pm \mathrm{SD})$. Al-Ghzawi \& Zaitoun (2008) crían reinas de las razas $A$. m. ligustica y $A$. $m$. syriaca en clima mediterráneo semiárido (Siria), encontrando un peso medio al nacimiento de $225,3 \mathrm{mg}$ en el caso de $A$. m. ligustica y $173,8 \mathrm{mg}$ para $A$. m. syriaca. Kahya 
et al. (2008) estudian el peso de reinas de la raza $A . m$. caucasica criadas artificialmente, buscando posibles correlaciones entre esta variable y el comportamiento reproductivo. Citan que el peso medio al nacimiento es de $193,3 \pm 1,51 \mathrm{mg}$, valor muy similar al obtenido por nosotros (194 \pm 26 mg). Okuyan \& Akyol (2018) encuentran que el peso al nacimiento de las reinas de la raza A. m. anatoliaca es de $166,39 \pm 1,83 \mathrm{mg}$, valor inferior al de otras razas de abejas.

Aunque no hemos encontrado en la bibliografía consultada ningún trabajo que estudie las posibles diferencias respecto del peso corporal de las reinas pertenecientes a diferentes razas, resulta evidente que existen ciertas diferencias que pueden ser significativas $\mathrm{y}$ tener base genética.

Hatjina et al. (2014) en su revisión de los métodos de valoración de la calidad de las abejas reinas, citan que una reina no debería pesar menos de $190 \mathrm{mg}$. Los pesos medios obtenidos por nosotros se resumen en la Tabla II, encontrando valores superiores a inferiores al límite de calidad fijado por estos autores.

En nuestro trabajo no evidenciamos un efecto diferenciador de la colmena o colonia criadora, en el peso de las reinas al nacimiento, ya que los análisis estadísticos no indican la existencia de diferencias significativas entre colmenas. En cambio, en el caso del peso de la celdilla si existen diferencias, siendo las abejas de la colmena 951 (ver Figura 1) las que fabricaron las celdillas utilizando una mayor cantidad de cera. Pero al no haber encontrado diferencias relacionadas con el peso de las reinas, podemos afirmar que probablemente el uso de una mayor cantidad de materia prima, no implicó que las larvas disfrutasen de unas mejores condiciones (ej. más espacio o alimento) en su desarrollo.

En el caso de que consideremos las fechas de cada ciclo de cría, sí existen diferencias significativas entre las colonias, estableciéndose subconjuntos que no podemos explicar con una hipótesis basada en un posible efecto temporal o climático. En el caso de la variable peso de las reinas la prueba a posteriori de K-W estableció dos subconjuntos, uno que incluía los ensayos en los que el peso de las reinas fue menor, y otro en el que dicho peso fue mayor; en el caso del peso de las celdillas se formaron tres subconjuntos.

Muy probablemente factores no identificados de tipo ambiental o biológico, como, por ejemplo, el estado nutricional o el sanitario, condicionaron estas diferencias.

Finalmente, si en el análisis estadístico consideramos cada fecha de forma independiente, y buscamos la existencia de diferencias significativas respecto al peso de los animales entre las colonias, la prueba de $\mathrm{K}-\mathrm{W}$ tampoco indica la existencia de diferencias significativas entre colmenas criadoras. Pensamos que los cambios en las condiciones ambientales que se producen en nuestra latitud entre los meses de marzo y mayo, condicionan de forma significativa las aptitudes para la cría de las colonias, pero no hemos encontrado ningún efecto significativo de la variable colmena en cada ciclo de cría.

\begin{tabular}{|c|c|c|c|c|c|c|c|}
\hline Ensayo & Colmena & $\begin{array}{l}\text { Peso reina } \\
\text { (mg) }\end{array}$ & $\begin{array}{l}\text { Peso celdilla } \\
\text { (mg) }\end{array}$ & Ensayo & Colmena & Peso reina $(\mathrm{mg})$ & Peso celdilla (mg) \\
\hline \multirow[t]{4}{*}{1} & 215 & $187 \pm 21$ & $620 \pm 17$ & \multirow[t]{4}{*}{5} & 215 & \multirow[t]{2}{*}{$187 \pm 06$} & \multirow[t]{2}{*}{$717 \pm 127$} \\
\hline & 326 & $200 \pm 07$ & $756 \pm 74$ & & 326 & & \\
\hline & 951 & $200 \pm 26$ & $758 \pm 47$ & & 951 & $190 \pm 07$ & $595 \pm 49$ \\
\hline & 991 & $220 \pm 35$ & $713 \pm 23$ & & 991 & $174 \pm 16$ & $641 \pm 40$ \\
\hline \multirow[t]{4}{*}{2} & 215 & $176 \pm 15$ & $658 \pm 44$ & \multirow[t]{4}{*}{6} & 215 & \multirow[t]{2}{*}{$203 \pm 13$} & \multirow[t]{2}{*}{$760 \pm 69$} \\
\hline & 326 & $186 \pm 11$ & $573 \pm 83$ & & 326 & & \\
\hline & 951 & $172 \pm 12$ & $763 \pm 82$ & & 951 & $170 \pm 06$ & $570 \pm 44$ \\
\hline & 991 & $170 \pm 23$ & $611 \pm 69$ & & 991 & $160 \pm 20$ & $577 \pm 81$ \\
\hline \multirow[t]{4}{*}{3} & 215 & $210 \pm 23$ & $511 \pm 33$ & \multirow[t]{4}{*}{7} & 215 & \multirow[t]{2}{*}{$191 \pm 25$} & \multirow[t]{2}{*}{$440 \pm 26$} \\
\hline & 326 & $223 \pm 55$ & $537 \pm 38$ & & 326 & & \\
\hline & 951 & $198 \pm 26$ & $687 \pm 73$ & & 951 & $180 \pm 28$ & $535 \pm 78$ \\
\hline & 991 & $203 \pm 18$ & $637 \pm 52$ & & 991 & $169 \pm 16$ & $436 \pm 40$ \\
\hline \multirow[t]{4}{*}{4} & 215 & $212 \pm 16$ & $744 \pm 63$ & \multirow[t]{4}{*}{8} & 215 & $214 \pm 18$ & $459 \pm 32$ \\
\hline & 326 & & & & 326 & $207 \pm 22$ & $437 \pm 52$ \\
\hline & 951 & & & & 951 & $215 \pm 31$ & $677 \pm 66$ \\
\hline & 991 & $193 \pm 22$ & $610 \pm 23$ & & 991 & $214 \pm 15$ & $470 \pm 42$ \\
\hline
\end{tabular}


Si en lugar de considerar el peso de las reinas, tenemos en cuenta la variable peso de la celdilla de cría, el análisis de $\mathrm{K}-\mathrm{W}$ indica la existencia de diferencias significativas entre colonias en los ensayos $1^{\circ}, 2^{\circ}, 3^{\circ}$, y $8^{\circ}$. La prueba K-W a posteriori en todos los casos muestra la existencia de dos subconjuntos homogéneos, variando los agrupamientos de las colmenas en cada caso. Aunque no podemos identificar una tendencia o patrón en el agrupamiento de las colmenas, en la mayoría de los ensayos es la colmena número 951 la que se diferencia del resto. Este resultado es coherente con los obtenidos en otros análisis ya que esta colonia es la que fabricaba las celdillas reales con una mayor cantidad de cera.

\section{CONCLUSION}

La eficiencia o rendimiento de un proceso de cría de reinas va a depender de un conjunto de factores de tipo biológico, ambiental y de manejo, siendo la tasa de aceptación un primer indicador que permite valorar el posible rendimiento. No hemos encontrado diferencias, respecto al peso al nacimiento de los insectos, ni desde un punto de vista general, ni entre colmenas dentro de un determinado ciclo de cría. Aunque no existen trabajos que aborden el estudio del peso al nacimiento de las reinas de diferentes razas, los datos encontrados en la bibliografía consultada nos llevan a pensar en su posible existencia.

\section{AGRADECIMIENTOS}

Esta investigación ha sido financiada por el Fondo Europeo de Desarrollo Regional (FEDER) 2014-2020, a través del Instituto Nacional de Investigación y Tecnología Agraria, y Alimentaria (INIA) y el proyecto RTA2017-00058-C04-04 Mejora de las condiciones de producción en explotaciones apícolas: desarrollo de procesos de descontaminación de cera, impacto de tratamientos veterinarios en gestión convencional y ecológica, e indicadores de bienestar de las colonias.

\section{BIBLIOGRAFÍA}

Al-Ghzawi A. \& S. Zaitoun (2008) Origin and rearing season of honeybee queens affect some of their physiological and reproductive characteristics. Entomological Research 38: 139-148

Cengiz M. M., K Yazici \& S. Arslan (2019) The effect of the supplemental feeding of queen rearing colonies on the reproductive characteristics of queen bees (Apis mellifera L.) Reared from egg and different old of larvae. Kafkas Universitesi Veteriner Fakultesi Dergisi 25: 849-855.

Delaney D. A., J. J. Keller, J. R. Caren, D. R. Tarpy 2011 . The physical, insemination, and reproductive quality of honey bee queens (Apis mellifera L.). Apidologie 42: 1-13.

Hatjina F., M. Bieńkowska, L. Charistos, R. Chlebo, C. Costa, M. M. Dražić, J. Filipi, A. Gregorc, E. N. Ivanova, N. Kezić, J. Kopernicky,
P. Kryger, M. Lodesani, V. Lokar, M. Mladenovic, B. Panasiuk, P. P. Petrov, S. Rašić, M. I. Smodis Sker, F. Vejsnæs, J. Wilde 2014. A review of methods used in some European countries for assessing the quality of honey bee queens through their physical characters and the performance of their colonies. Journal of Apicultural Research 53: 337-363.

Kruk C. \& W. Skowronek (2002). Effect of different factors on the efficiency of honey bee queen rearing. Journal of Apiculture Science 46: 41-49.

Mirza, E., M. Dragan \& S. Sherbanescus 1967. Seasonal variability in the weight of emerging queens. Procedings of XXIst. International Apicultural Congress. Romania 269-273.

Mohammedi A. \& Y. Le Conte (2000). Do environmental conditions exert an effect on nest-mate recognition in queen rearing honey bees? Insectes Sociaux 47: 307-312.

Kahya Y., H V. Gençer \& J. Woyke 2008. Weight at emergence of honey bee (Apis mellifera caucasica) queens and its effect on live weights at the pre and post mating periods. Journal of Apicultural Research 47: 118-125.

Okuyan S. \& E. Akyol 2018. The effects of age and number of grafted larvae on some physical characteristics of queen bees and acceptance rate of queen bee cell. Turkish Journal of Agriculture - Food Science and Technology 6: 1556-1661.

Rangel J., J. J. Keller \& D. R. Tarpy 2012. The effects of honey bee (Apis mellifera L.) queen reproductive potential on colony growth. Insectes Sociaux 60: 65-73.

Honey bees consider larval nutritional status rather than genetic relatedness when selecting larvae for emergency queen rearing

Sagili R. R., B. N. Metz, H. M. Lucas, P. Chakrabarti, C. R. Breece 2018 Honey bees consider larval nutritional status rather than genetic relatedness when selecting larvae for emergency queen rearing. Scientific Report 8: 7679.

Skowronek W., M. Bienkowska, C. Kruk 2004. Changes in Body Weight of Honeybee Queens during their maturation. Journal of Apicultural Science 48: 61-67.

Wilkinson D. \& M. A. Brown 2002. Rearing queen honey bees in a queeright colony. American Bee Journal 142: 270-274.

Winston M. L. 1991. The Biology of the Honey Bee. Harvard University Press, Cambridge, Massachusetts.

Woyke J. 1971. Correlations between the age at which honey bee brood was grafted, characteristic of resultant queens and result of insemination. Journal of Apicultural Research 10: 45-55. 